\title{
Endoscopic Ultrasound-Guided Liver Biopsy: Which Needle Is the Best?
}

\author{
Suhang Verma ${ }^{1}$ Surinder S. Rana ${ }^{1}$ \\ ${ }^{1}$ Department of Gastroenterology, Post Graduate Institute of \\ Medical Education and Research (PGIMER), Chandigarh, India
}

J Digest Endosc 2020;10:242-244

Evaluation of the liver histology on the specimen obtained by liver biopsy forms an indispensable part of diagnosis and management of many parenchymal liver disorders despite the advances in noninvasive modalities. ${ }^{1}$ Apart from the diagnostic role, it is also crucial in staging and prognosticating various liver disorders with a vast list of established and expanding indications. ${ }^{2}$ Since the advent of percutaneous liver biopsy the route, technique, and modalities available for liver biopsy have undergone many changes. ${ }^{3}$ While conventionally performed in a blind fashion through percutaneous route, the inherent limitations and complications of this technique necessitated emergence of new alternative techniques including image-guided biopsy (ultrasound, fluoroscopy, computed tomography, and magnetic resonance imaging), transjugular liver biopsy, and laparoscopic liver biopsy. ${ }^{4}$ Most liver biopsies in the current era are performed via percutaneous route (almost always under radiological guidance) and often as an outpatient procedure. Pain remains one of the most common complication of percutaneous liver biopsy although it is usually mild in majority of the patients. ${ }^{5}$ The extensive experience with percutaneous liver biopsy has demonstrated it to be largely a safe procedure with a 0 to $0.7 \%$ risk of major complications and 0 to $0.5 \%$ risk of mortality usually because of torrential bleed. ${ }^{1}$

Although the image-guided percutaneous liver biopsy has stood the test of time, certain situations (clinically demonstrable ascites; bleeding diathesis; small, cirrhotic liver; morbid obesity) render it nonfeasible and/or increase the complication rates. Transvenous (transjugular) liver biopsy is often the preferred technique for tissue acquisition in such a scenario. ${ }^{1,6}$ In experienced hands, transjugular biopsy has been shown to have a high technical success (96.8\%) with a small rate of major complications $(0.56 \%)$ and mortality $(0.09 \%)^{7}$ Despite being a safe technique, availability of an experienced interventional radiologist and support staff, use of ionizing radiation, nonfeasibility in hepatic vein/inferior vena cava thrombosis, lack of targeted sampling, risk of vascular or biliary injury, and sometimes insufficient yield are some of the important limitations of transjugular liver biopsy. ${ }^{8}$

\begin{abstract}
Address for correspondence Surinder Singh Rana, MD, DM, Department of Gastroenterology, Post Graduate Institute of Medical Education and Research (PGIMER), Chandigarh 160 012, India (e-mail: drsurinderrana@gmail.com).
\end{abstract}

Endoscopic ultrasound-guided liver biopsy (EUS-LB) is a relatively new and emerging modality for acquiring hepatic tissue. ${ }^{9}$ Within a short span of time it has been established as a safe alternative to traditionally available methods. Compared with the previously discussed techniques, EUS-LB offers multiple advantages including real-time, high-resolution image guidance, visualization of the needle tract during entire procedure, ability to target both lobes of liver, reduced pain and apprehension, better patient comfort, and shorter hospital stay. ${ }^{10-13}$ Additionally, it also provides opportunity to measure the portal venous pressure and perform elastography during the same session. ${ }^{14,15}$ EUS-LB can also be feasible in patients with morbid obesity and ascites where percutaneous biopsy is contraindicated. ${ }^{13}$ Furthermore, it confers an opportunity to perform liver biopsy in the same session if another endoscopic procedure is already warranted (esophagogastroscopy or EUS). Studies have demonstrated a high technical success and adequacy of tissue yield (>98\%) with EUS-LB that are comparable to the percutaneous or transjugular routes. ${ }^{12,16,17}$ In a recently published meta-analysis including 9 studies (437 patients) the pooled rate of histological diagnosis was $93.9 \%$ (95\% confidence interval $[\mathrm{CI}]=84.9-97.7$, heterogeneity $\left.\left[{ }^{2}\right]=75.3 \%\right) .{ }^{18}$ Despite the high yield the rates of adverse events remain low. In the same meta-analysis, pooled overall adverse event rates were $2.3 \%$ $\left(95 \% \mathrm{CI}=1.1-4.8, I^{2}=0\right)$ and the risk of bleeding was $1.2 \%$ $\left(95 \% \mathrm{CI}=0.4-3.7, I^{2}=0\right) .{ }^{18}$ Despite the advantages and proven efficacy and safety, EUS-LB remains underutilized due to concerns regarding adequacy of tissue obtained, the problem of tissue fragmentation, cost of needles/anesthesia, and fear of bleeding.

Since its inception, various needle types have been used for performing EUS-LB. Initial studies in porcine models and humans using "tru-cut" needles yielded insufficient samples and procedure was technically challenging. ${ }^{19,20}$ With the development of new and improved needles, including fine needle biopsy (FNB) needles, use of "tru-cut" needle has fallen out of practice. 19G EUS-FNA (fine needle aspiration) "non-tru-cut" needle was first used for EUS-LB in 2012. ${ }^{21}$ Since then, several needle types (19G FNA, 19G FNB, and 22G
License terms

()(1) $\Theta \circledast$ 
FNB) have been evaluated in prospective and retrospective studies with variable tissue yields and fragmentation rates. ${ }^{11}$ Similarly, different biopsy techniques have been developed in attempts to further increase the yield and reduce tissue fragmentation, including "dry suction," "dry heparin," and "wet suction." 22 There are limited prospective studies available offering direct comparison between needle types and sizes. Mok et al compared 19G FNA needle with 22G FNB needle in a prospective crossover study. ${ }^{23}$ Although preprocessing adequacy of sample was comparable between both the groups, the rates of tissue fragmentation were significantly higher in $22 \mathrm{G}$ group due to smaller core size. Therefore, samples adequate for pathological interpretation could be obtained in only $60 \%$ cases (compared with $90 \%$ with $19 \mathrm{G}$ needle) and there was no additional safety benefit with smaller needle. ${ }^{11,23}$ However, due to lack of adequate prospective randomized trials for direct comparison of needle types, size, and techniques, there is no consensus yet regarding the best needle for performing EUS-LB. In this edition of "News" we discuss two recently published articles exploring this important issue.

The first study is an interesting single-center, prospective, randomized-controlled trial by Ching-Companioni et al, published in Endoscopy. ${ }^{24}$ The group from United States conducted a trial between October 2017 and December 2017, comparing the tissue yield and adequacy of 19G FNA (Expect Flexible 19G, Boston Scientific, Marlborough, Massachusetts, USA; $n=20$ ) and 19G FNB Franseen tip needle (Acquire $19 \mathrm{G}$, Boston Scientific, Marlborough, Massachusetts, USA; $n=20$ ). The EUS-LB was performed using linear echoendoscope under propofol sedation. "Wet-suction" method of biopsy was used wherein 2 to $3 \mathrm{~mL}$ of heparin (100 units $/ \mathrm{mL}$ ) was flushed through the needle and both the lobes of liver were sampled (one pass each) with each pass comprising of 7 to 10 to-and-fro needle movements using fanning technique. After extracting, the samples were processed and cores were macroscopically and microscopically analyzed pre and post processing.

The demographic profile of both groups was comparable and the most common indication was evaluation of abnormal liver enzymes ( $n=32,80 \%$ ). Majority ( $n=24,60 \%$ ) of patients studied were obese and the procedure was successful in all 40 patients. On comparing the primary outcome (mean preprocessing length of the longest core of liver biopsy obtained) between the two groups, the mean length was significantly higher in the FNB group as compared with the FNA group $(2.09 \pm 0.41 \mathrm{~cm}$ vs. $1.47+0.46 \mathrm{~cm}, p$-value $<0.001)$. Most of the other secondary outcomes also appeared to favour EUS-FNB needle. The mean postprocessing length of longest core was also significantly higher in the FNB group (1.78 \pm $0.66 \mathrm{~cm}$ vs. $1.05 \pm 0.42 \mathrm{~cm}, p$-value $<0.001$ ) and so were the mean aggregate preprocessing and postprocessing lengths $(15.78 \pm 5.19 \mathrm{~cm}$ vs. $10.89 \pm 4.38 \mathrm{~cm}, p$-value $0.003 ; 15.32 \pm$ $5.24 \mathrm{~cm}$ vs. $11.4 \pm 5.55 \mathrm{~cm}, p$-value 0.028 , respectively). An intact specimen longer than $2 \mathrm{~cm}$ was obtained in $10(50 \%)$ patients in the FNB group as compared with only $3(15 \%)$ patients in the FNA group ( $p$-value 0.04). On microscopic examination, FNB yielded a significantly higher number of mean complete portal triads in specimen ( $42.6 \pm 25$ vs. 18.1 $\pm 9.3, p$-value $<0.001)$. Although $18(90 \%)$ patients in the FNB group had 11 or more complete portal tract triads in specimen (minimal recommendation for adequacy for liver biopsy) as compared with 14 (70\%) patients in the FNA group, the difference could not reach statistical significance $(p$-value $=0.24) .{ }^{1}$ Despite underperformance of FNA needle, pathological diagnosis could be reached in all the patients in both groups. The only adverse event seen in study was pain, developing in 7 (35\%) patients in the FNB group and 8 (40\%) patients in the FNA group ( $p$-value $=0.74)$. No serious adverse events were encountered and all patients were discharged on the same day. The authors concluded that 19G FNB needle is superior to 19G FNA needle in delivering longer, intact specimens with reduced fragmentation and higher yield of complete portal triads with a favorable safety profile.

The other study that we are discussing is a prospective study to evaluate the efficacy and safety of a smaller 22G EUS-FNB needle for EUS-LB that has been published by Hasan et al from United States in Endoscopy. ${ }^{25}$ This study is a single-center, open-label, prospective trial conducted between August 2017 and June 2018 in 40 patients who underwent EUS-LB using a 22G EUS-FNB Franseen needle (Acquire, Boston Scientific, Marlborough, Massachusetts, USA). The EUS-LB was performed with a linear echoendoscope and both the lobes of liver were sampled with three passes (two passes for left lobe and one pass for right lobe). No suction was used and each pass comprised 3 to 4 to-and-fro movements with a fanning technique. A preprocessing onsite macroscopic assessment of the specimen was made, followed by postprocessing macroscopic and microscopic assessment.

The median age of patients was 61 years, (interquartile range $[\mathrm{IQR}] 21.5)$ and 14 (35\%) were males. All (100\%) patients underwent EUS-LB biopsy for elevated liver enzymes and a total of 120 passes were made in 40 patients. In two patients, the right lobe could not be sampled due to surgically altered anatomy, while both lobes were sampled in the remaining 38 (95\%). The primary outcome was diagnostic adequacy of sample obtained-adequacy of gross specimen (presence of at least one core fragment and aggregate core length $\geq 15 \mathrm{~mm}$ ) and adequacy of diagnostic yield (sufficient material for pathologist to reach a diagnosis). On visual examination, 119/120 (99.2\%) passes yielded adequate gross tissue sample (39 [97.5\%] patients in the first pass while all 40 [100\%] patients within two passes). The median longest and aggregate lengths of specimen from both the lobes were comparable (left lobe $12 \mathrm{~mm}$ [IQR $6.25 \mathrm{~mm}$ ] and $20 \mathrm{~mm}$ [IQR $11 \mathrm{~mm}$ ], respectively; right lobe $11 \mathrm{~mm}$ [IQR $5.75 \mathrm{~mm}$ ] and $20 \mathrm{~mm}$ [IQR $11.7 \mathrm{~mm}$ ], respectively). The median cumulative core length per patient was $55 \mathrm{~mm}$ (IQR $24.5 \mathrm{~mm}$ ), with a median core thickness of $0.1 \mathrm{~mm}$. The median number of complete portal tracts per pass for left and right lobes were 14 (IQR 11) and 15 (IQR 9.25), respectively, while the median cumulative number of complete portal triad per patient was 42 (IQR 24.5). The adequacy of samples for reaching accurate histological diagnosis was $100 \%$ but there was presence of moderate amount of tissue distortion post processing. As in previous study, all the patients were discharged on the same 
day. The adverse events (secondary outcome) included mild abdominal pain in $6(15 \%)$ patients, self-resolving fever in one patient, and unexplained death within 24 hours of procedure in one patient. This study demonstrated that it is feasible to obtain an adequate liver biopsy specimen using 22G EUS-FNB needle with high success rates and despite some distortion during processing, diagnostic accuracy is high.

\section{Commentary}

EUS-LB is an emerging and underutilized modality for liver tissue sampling with many inherent advantages. The safety of the procedure is well established. However, procedure and technique is still evolving in an attempt to increase the yield and diagnostic accuracy. The most commonly used needle at present is $19 \mathrm{G}$ needle and $19 \mathrm{G}$ FNB appears to have a definite superiority over 19G FNA needle in terms of the yield. Emerging data suggest that a smaller 22G FNB needle may also have an adequate diagnostic yield although head-to-head comparison with 19G FNB needle is needed and in the next few years we will probably have the definitive answer to this clinical problem.

\section{Financial Disclosures \\ None.}

\section{Conflict of Interest}

None declared.

\section{References}

1 Rockey DC, Caldwell SH, Goodman ZD, Nelson RC, Smith AD; American Association for the Study of Liver Diseases. Liver biopsy. Hepatology 2009;49(3):1017-1044

2 Sheela H, Seela S, Caldwell C, Boyer JL, Jain D. Liver biopsy: evolving role in the new millennium. J Clin Gastroenterol 2005;39(7):603-610

3 Bingel A. Ueber die parenchympunktion der leber. Verh Dtsch Ges Inn Med 1923;35:210-212

4 Grant A, Neuberger J; British Society of Gastroenterology. Guidelines on the use of liver biopsy in clinical practice. Gut 1999;45(Suppl 4):IV1-IV11

5 Eisenberg E, Konopniki M, Veitsman E, Kramskay R, Gaitini D, Baruch Y. Prevalence and characteristics of pain induced by percutaneous liver biopsy. Anesth Analg 2003;96(5):13921396 table of contents

6 Behrens G, Ferral H. Transjugular liver biopsy. Semin Intervent Radiol 2012;29(2):111-117

7 Kalambokis G, Manousou P, Vibhakorn S, et al. Transjugular liver biopsy-indications, adequacy, quality of specimens, and complications-a systematic review. J Hepatol 2007;47(2):284-294

8 Dohan A, Guerrache Y, Boudiaf M, Gavini JP, Kaci R, Soyer P. Transjugular liver biopsy: indications, technique and results. Diagn Interv Imaging 2014;95(1):11-15
9 Gleeson FC, Clayton AC, Zhang L, et al. Adequacy of endoscopic ultrasound core needle biopsy specimen of nonmalignant hepatic parenchymal disease. Clin Gastroenterol Hepatol 2008;6(12):1437-1440

10 Diehl DL. Endoscopic ultrasound-guided liver biopsy. Gastrointest Endosc Clin N Am 2019;29(2):173-186

11 Mok SR, Diehl DL. The role of EUS in liver biopsy. Curr Gastroenterol Rep 2019;21(2):6

12 Diehl DL, Johal AS, Khara HS, et al. Endoscopic ultrasound-guided liver biopsy: a multicenter experience. Endosc Int Open 2015;3(3):E210-E215

13 Shah AR, Al-Hanayneh M, Chowdhry M, Bilal M, Singh S. Endoscopic ultrasound guided liver biopsy for parenchymal liver disease. World J Hepatol 2019;11(4):335-343

14 Schulman AR, Lin MV, Rutherford A, Chan WW, Ryou M. A prospective blinded study of endoscopic ultrasound elastography in liver disease: towards a virtual biopsy. Clin Endosc 2018;51(2):181-185

15 Samarasena JB, Yu AR, Chang KJ. EUS-guided portal pressure measurement (with videos) Endosc Ultrasound 2018;7(4):257-262

16 Pineda JJ, Diehl DL, Miao CL, et al. EUS-guided liver biopsy provides diagnostic samples comparable with those via the percutaneous or transjugular route. Gastrointest Endosc 2016;83(2):360-365

17 Shuja A, Alkhasawneh A, Fialho A, et al. Comparison of EUSguided versus percutaneous and transjugular approaches for the performance of liver biopsies. Dig Liver Dis 2019;51(6):826-830

18 Mohan BP, Shakhatreh M, Garg R, Ponnada S, Adler DG. Efficacy and safety of EUS-guided liver biopsy: a systematic review and meta-analysis. Gastrointest Endosc 2019;89(2):238-246.e3

19 Wiersema MJ, Levy MJ, Harewood GC, Vazquez-Sequeiros E, Jondal ML, Wiersema LM. Initial experience with EUS-guided trucut needle biopsies of perigastric organs. Gastrointest Endosc 2002;56(2):275-278

20 Gleeson FC, Levy MJ. EUS Trucut biopsy liver parenchyma acquisition and yield are comparable to that of a transjugular liver biopsy. Gastrointest Endosc 2009;70(5):1046, author reply $1046-1047$

21 Stavropoulos SN, Im GY, Jlayer Z, et al. High yield of same-session EUS-guided liver biopsy by 19-gauge FNA needle in patients undergoing EUS to exclude biliary obstruction. Gastrointest Endosc 2012;75(2):310-318

22 Mok SR, Diehl DL, Johal AS, et al. A prospective pilot comparison of wet and dry heparinized suction for EUS-guided liver biopsy (with videos) Gastrointest Endosc 2018;88(6):919-925

23 Mok SR, Diehl DL, Johal AS, et al. Mo1245 19 versus 22 gauge fine needle biopsy for endoscopic ultrasound guided liver biopsy (EUS-LB): a prospective randomized trial. Gastrointest Endosc 2017;85(5):AB473-AB4

24 Ching-Companioni RA, Diehl DL, Johal AS, Confer BD, Khara HS. 19G aspiration needle versus 19G core biopsy needle for endoscopic ultrasound-guided liver biopsy: a prospective randomized trial. Endoscopy 2019;51(11):1059-1065

25 Hasan MK, Kadkhodayan K, Idrisov E, et al. Endoscopic ultrasound-guided liver biopsy using a 22-G fine needle biopsy needle: a prospective study. Endoscopy 2019;51(9):818-824 